Paper Number 01ICES-2372

\title{
Shuttle Spacesuit: Fabric/LCVG Model Validation
}

J.W. Wilson

NASA Langley Research Center, Hampton, VA 23681-2199

J.Tweed

Old Dominion University, Norfolk, VA 23508

C. Zeitlin

DOE Lawrence Berkeley National Laboratory, Berkeley, CA 94720

M.-H. Y. Kim

College of William and Mary, Williamsburg, VA 23185

B.M. Anderson

George Washington University, Hampton, VA 23681

F.A. Cucinotta

NASA Johnson Space Center, Houston, TX 77058

J. Ware, A.E.Persans

ILC Dover, Frederica, DE 19946-2000

Copyright @ 2001 Society of Automotive Engineers, Inc.

\begin{abstract}
A detailed spacesuit computational model is being developed at the Langley Research Center for radiation exposure evaluation studies. The details of the construction of the spacesuit are critical to estimation of exposures and assessing the risk to the astronaut on EVA. Past evaluations of spacesuit shielding properties assumed the basic fabric lay-up (Thermal Micrometeroid Garment, fabric restraints, and pressure envelope) and Liquid Cooling and Ventilation Garment (LCVG) could be homogenized as a single layer overestimating the protective properties over 60 percent of the fabric area. The present spacesuit model represents the inhomogeneous distributions of LCVG materials (mainly the water filled cooling tubes). An experimental test is performed using a 34-MeV proton beam and highresolution detectors to compare with model-predicted transmission factors. Some suggestions are made on possible improved construction methods to improve the spacesuit's protection properties.
\end{abstract}

\section{INTRODUCTION}

The construction of the International Space Station requires 1500 hours of extravehicular activity (EVA) during construction and 400 hours per year in operations and maintenance. The orbit at 51.6 degrees inclination is in a highly variable radiation environment driven by solar activity. Solar particle events will enter this region, especially during an associated geomagnetic storm. The geomagnetic storm conditions also increase the trapped electron environment by up to four orders of magnitude; this electron enhancement can persist for several days. Although safety demands that such events be avoided if possible, work activity may not allow the astronaut to seek shelter in a timely fashion and the transmission properties of the basic suit are critical to astronaut safety. These exposures will add to the usual quite-time exposures to trapped protons and electrons and galactic cosmic rays experienced by the astronaut.

The driving factor in this study is the need for an adequate spacesuit model for the estimation of exposures for mission planning and evaluation of safety during radiation field disturbance. Although there are several issues related to the protective properties of the spacesuit, we will address herein only the least shielded portions of the suit, which provides the basic protection of a large fraction of the body surface. In particular, the basic fabric portion of the suit mainly gives protection to the skin from the least penetrating radiation components. 
Other critical spacesuit issues will be addressed in subsequent studies. In addition, an improved understanding of the basic transmission properties of the spacesuit fabric components, which is a complex lay-up of materials, will provide a guide to improving the basic fabric design. Other critical spacesuit components will be addressed in subsequent studies.

It is customary in estimating the fabric transmission properties to assume the basic fabric lay-up is a homogenized whole of the associated materials [1,2]. In the present document we will analyze this assumption in detail and perform experimental tests of this assumption using low-energy proton beams.

\section{COMPUTATIONAL MODEL}

The types and energy distributions of particles transmitted through a shield material require the solution to the Boltzmann transport equation with appropriate boundary conditions related to the external space radiation environment. The relevant transport equation [3] for the flux density $\phi_{j}(\boldsymbol{x}, \boldsymbol{\Omega}, E)$ of type $j$ particles moving in direction $\boldsymbol{\Omega}$ with energy $E$ is given as

$$
\begin{aligned}
& \boldsymbol{\Omega} \bullet \nabla \phi_{j}(\boldsymbol{x}, \boldsymbol{\Omega}, E)= \\
& \sum \int \sigma_{j k}\left(\boldsymbol{\Omega}, \boldsymbol{\Omega}^{\prime}, E, E^{\prime}\right) \phi_{k}\left(\mathbf{x}, \boldsymbol{\Omega}^{\prime}, E^{\prime}\right) d \boldsymbol{\Omega}^{\prime} d E^{\prime} \\
& -\sigma_{j}(E) \phi_{j}(\mathbf{x}, \boldsymbol{\Omega}, E)
\end{aligned}
$$

where $\sigma_{j}(E)$ is the media macroscopic cross section for removal of $j$ particles of energy $E, \sigma_{j k}\left(\Omega, \Omega, \Omega^{\prime}, E, E^{\prime}\right)$ are the media macroscopic cross sections for various atomic and nuclear processes adding $j$ particles of energy $E$ in direction $\boldsymbol{\Omega}$ including spontaneous disintegration. In general, there are hundreds of particle fields $\phi_{j}(\boldsymbol{x}, \boldsymbol{\Omega}, E)$ with several thousand cross-coupling terms $\sigma_{j k}\left(\Omega, \Omega^{\prime}, E, E^{\prime}\right)$ through the integral in equation (1). The total cross section $\sigma_{j}(E)$ with the medium for each particle type of energy $E$ may be expanded as

$$
\sigma_{j}(E)=\sigma_{j, a t}(E)+\sigma_{j, e l}(E)+\sigma_{j, r}(E)
$$

where the first term refers to collision with atomic electrons, the second term is for elastic nuclear scattering, and the third terms describes nuclear reactive processes and is ordered as $1: 10^{-5}: 10^{-8}$. This ordering allows flexibility in expanding solutions to the Boltzmann equation as a sequence of physical perturbative approximations. The atomic interactions are treated using energy moments in which the leading term is the usual continuous slowing down approximation. Special problems arise in the perturbation approach for neutrons for which the nuclear elastic process appears as the firstorder perturbation and has been the recent focus of research $[4,5]$.
Important to low-energy proton penetration are the atomic processes. Traditionally we have used the first energy moment to describe the energy loss in atomic processes and the resulting range-energy relations. More recently, the second energy moment has been added as related to range straggling [6] with added improvements resulting to the evaluations shown in fig. 1. Shown in the figure is the second moment presented as the full width at half maximum (FWHM) of the resultant energy distribution about the first energy moment seen at a depth $\mathrm{x}$ in aluminum for $49.1 \mathrm{MeV}$ protons where $R_{0}$ is the mean range. Excellent agreement is found for the second energy moment in comparison with the experiments of Tschalar and Macabee [7].

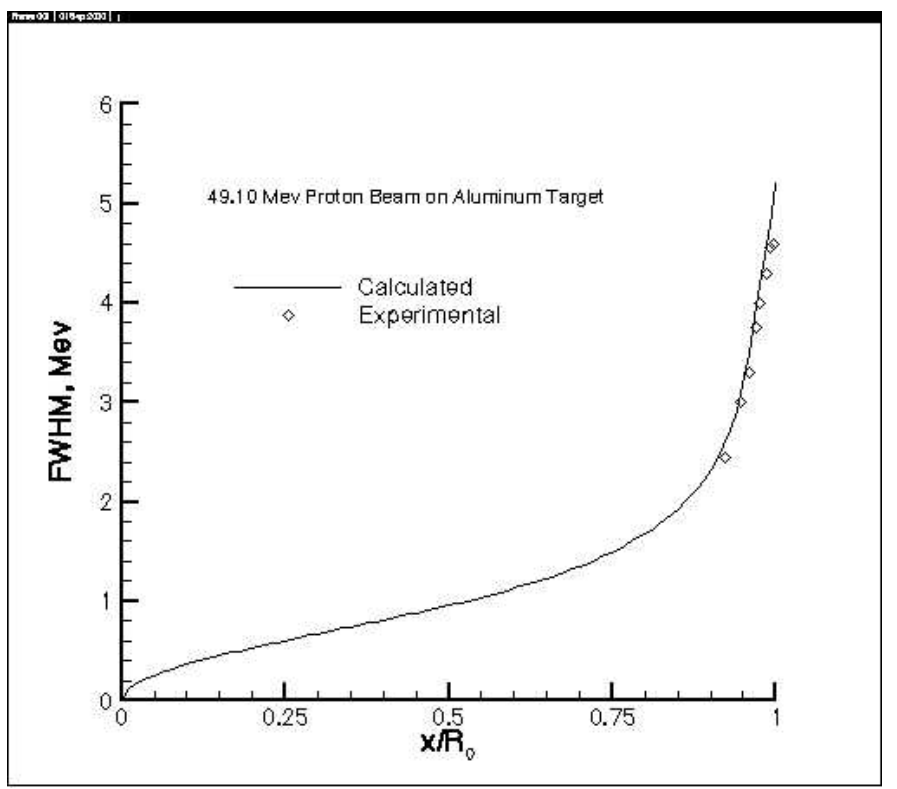

Fig.1 FWHM of $49.1 \mathrm{MeV}$ protons in an aluminum

target.

\section{SPACESUIT FABRIC MODEL}

The Shuttle spacesuit is manufactured by ILC Dover for use in the space program. The basic components are shown in fig. 2. Not shown in the figure is the life support system held mainly in a backpack attached to the Hard Upper Torso (HUT). We are mainly concerned herein with the Arm assembly and Lower Torso Assembly (LTA) consisting mainly of fabric and the Liquid Cooling and Ventilation Garment (LCVG) consisting of fabric and the water filled cooling tubes. The basic lay-up of the fabric and LCVG are shown in fig. 3.

The most easily penetrated portion of the spacesuit is covered only by the fabric (Arm assembly and LTA) and the LCVG. This is of greatest importance when the environment contains low energy particles with limited penetration power. Most environmental components contain such particles and are often the most intense component. They are only of concern for tissues which are poorly shielded and not of concern within a space vehicle assembly such as the Shuttle or ISS or for organs 
deep within the body. The basal layer of the skin is somewhat sensitive to radiation and therefore of concern in a lightly shielded spacesuit in an intense and lowenergy environment.

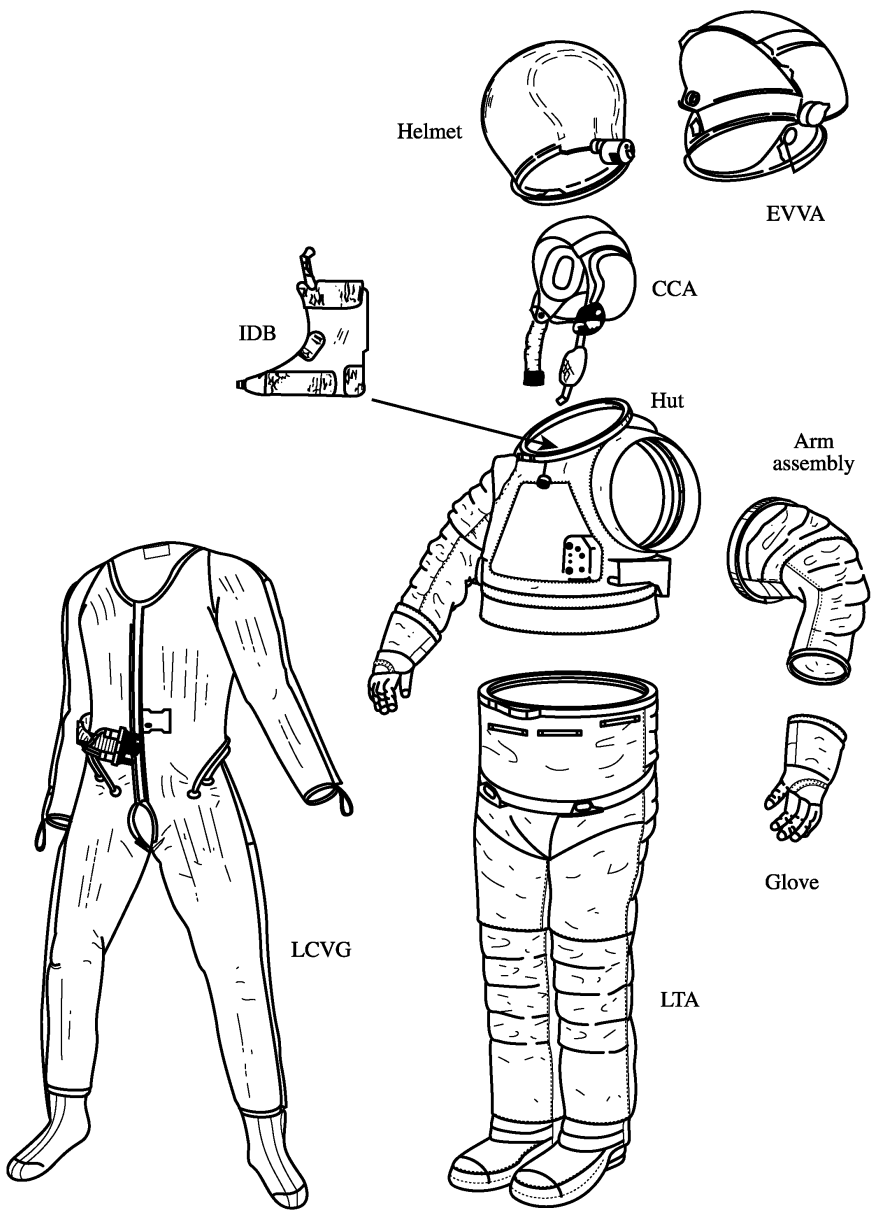

Fig. 2. Basic components of the Shuttle spacesuit.

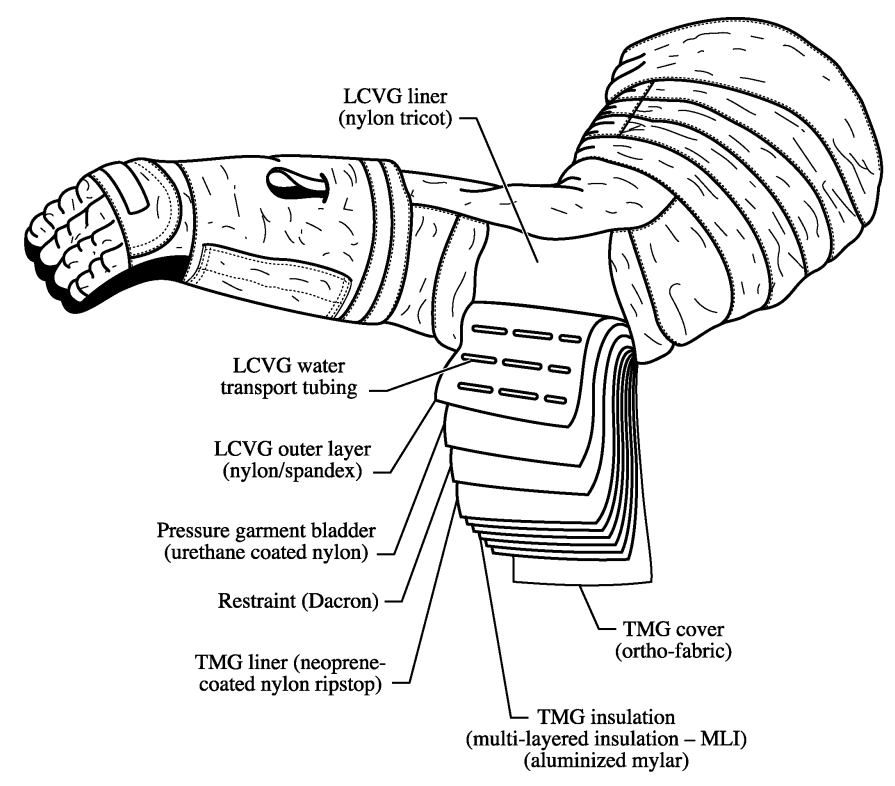

Fig. 3. Cross section of material lay-up.
The material lay-up for the fabric including the inner LCVG is given in table 1 . The outer fabric layer, the Thermal Micrometeroid Garment (TMG)is composed of the OrthoFabric, five layers of Reinforced Aluminized Mylar for thermal insulation and Neoprene Coated Nylon Ripstop. Below the TMG is the Dacron ${ }^{\circledR}$ pressure restraint. This is followed by the Urethane Coated Nylon (pressure bladder) and the LCVG of a multifilament Nylon/Spandex knit which contains, the ethylvinyl-acetate tubes filled with water.

Table 1. Material lay-up of the spacesuit fabric and water filled tube $[1,8]$.

\begin{tabular}{|l|l|}
\hline Material & $\begin{array}{l}\text { Areal density, } \\
\mathrm{g} / \mathrm{cm}^{2}\end{array}$ \\
\hline Orthofabric-Teflon/Nomex/Kevlar & 0.049 \\
Reinforced Aluminized Mylar & 0.014 \\
Neoprene Coated Ripstop & 0.028 \\
Dacron® Polyester & 0.021 \\
Urethane Coated nylon Ripstop & 0.014 \\
Nylon/Spandex/water/ethylvinylacetate & 0.154 \\
\hline
\end{tabular}

In past calculations [1], the materials in table 1 were converted into equivalent amounts of aluminum by scaling with the ratio of range of a $50 \mathrm{MeV}$ proton in aluminum to the range in the specific material and calculations made for penetration in the equivalent aluminum. There are three concerns with this approach: the equivalent aluminum scale factor depends on the proton energy and the assumed $50 \mathrm{MeV}$ is not accurate for estimation for minimum penetration particles, the water filled tubes cover only 40 percent of the surface area and the homogenization with the Spandex overestimates the fabric (with homogenized tube) protection properties over 60 percent of the area, and many components of the fabric lay-up are inhomogeneous structures and may not be well represented by an average areal density.

The surest way to represent the actual fabric/tube transmission properties is to remove these defects by representing the water filled tube geometry specifically, transporting through actual material layers as opposed to assuming equivalent aluminum, and performing penetration test to test models of the inhomogeneities within the remaining fabric. These tasks are performed in the present study and the fabric transmission properties are represented as an analytical model with good agreement with low-energy proton transmission testing. The improved understanding of the fabric transmission properties will allow redesign considerations to improve the spacesuit radiation safety. The basic penetration test is given in fig. 4 . The test is in principle quite simple; a low-energy proton beam is incident from the left on a swatch of the spacesuit and 
water filled tubes as shown. The arrangement of the experimental setup is shown in fig. 5 as used in the present testing. A reasonably uniform beam of approximately $34.5 \mathrm{MeV}$ protons enters the Lexan collimator from the left and is monitored by a $3 \mathrm{~mm}$ thick silicon detector $(\mathrm{d} 3 \mathrm{~mm} 1)$. The transmitted spectrum through d3mm1 was measured in a "target out" test. The monitored beam passes through the target station and analyzed by a set of position sensitive detectors (PSD1X\&Y) with total remaining energy detected by the 5-mm silicon detector ( $\mathrm{d} 5 \mathrm{~mm} 1)$.

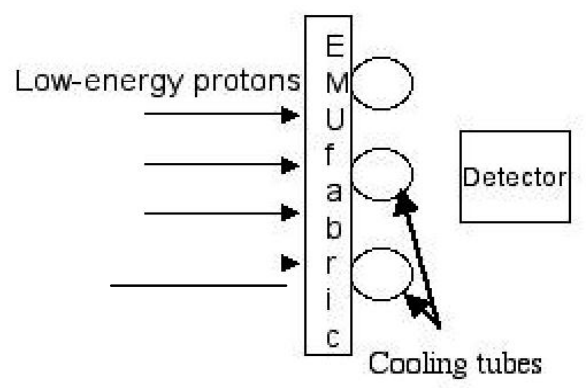

\section{Planned penetration testing}

Fig. 4. Basic experimental set up.

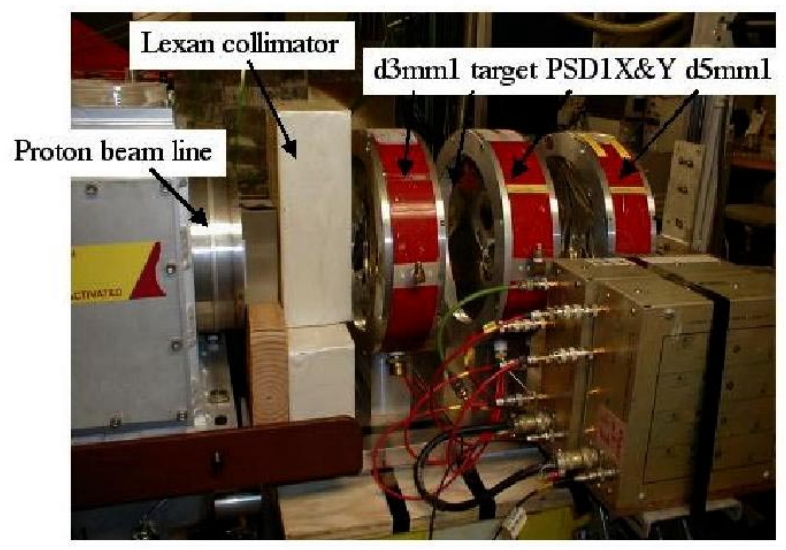

Fig. 5. Proton beamline used at the LBNL 88" cyclotron.

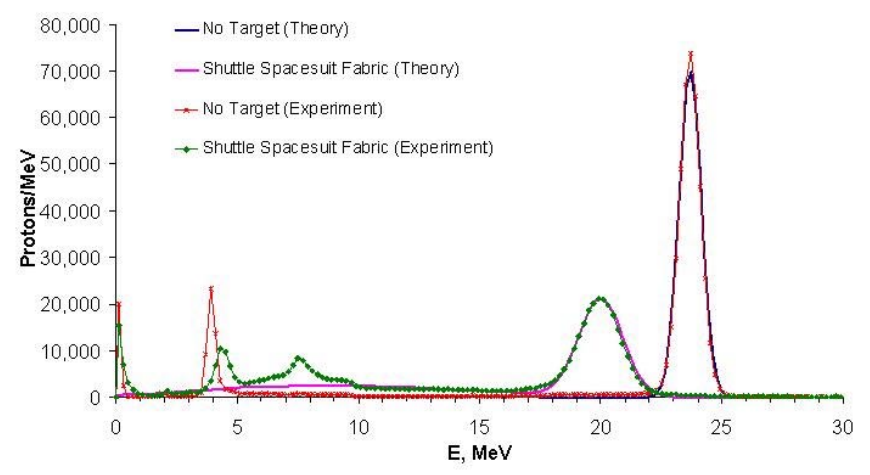

Fig. 6. Experimental and analysis results.

\section{RESULTS AND IMPLICATIONS}

The beam was first analyzed without the target in place with results (no target) as the near normal distribution of particles at the far right in fig. 6. The computational model was fit to the beam parameters before the $\mathrm{d} 3 \mathrm{~mm} 1$ and found to be of $34.54 \mathrm{MeV}$ with a standard deviation of $0.27 \mathrm{MeV}$ resulting in the 23.68 $0.46 \mathrm{MeV}$ beam incident on the target shown in the figure. The additional broadening of the energy spectrum is due to straggling in the $\mathrm{d} 3 \mathrm{~mm} 1$ monitor. The results of the analysis are shown in comparison to the experimental estimate of the beam properties at the target. There is an added spectral feature near $4 \mathrm{MeV}$ in the experimental data believed to be due to multiple scattering effects in the collimator.

The beam transmitted through the target is shown also in the figure. The spectral feature at $19.94 \pm 0.967 \mathrm{MeV}$ results from the transmission through the fabric without a tube in the proton path. The increased width of this peak relative to the beam before the target results from straggling effects in the fabric and the non-uniformity of some of the fabric components. The fabric is best fit as a normal distribution of material of mean thickness of $0.161 \pm 0.03 \mathrm{~g} / \mathrm{cm}^{2}$ of material. The mean areal density of the fabric (without the cooling tubes) is measured to be $0.185 \mathrm{~g} / \mathrm{cm}^{2}$. The height of the transmitted peak is critically dependent on the size of the tube (tube diameter is $4 \mathrm{~mm}$ ) and tube spacing (1 per centimeter). In the model, the water filled tube was assumed to be a homogeneous mixture of appropriate mass of water and ethylvinylacetate. The experiment indicates that the improved penetration properties of the water may be apparent in the data as the experimental transmitted spectral feature near $7 \mathrm{MeV}$ may be due to the inner filling of water in the tube. Hence some added detail for the model is required.

\section{FUTURE ACTIVITY}

The next step in the analysis will be to model the tube to better understand the lowest energy transmission of this complex system. Additional experiments will be performed to better understand the source of the nonuniformity of the fabric components. This will be accomplished by noting that the penetration properties of the fabric components are near that of polycarbonate. The nonuniform elements (e.g., spandex) will be replaced by an equivalent sheet of uniform polycarbonate and the transmitted spectrum determined and analyzed. Following this activity should be a reasonable model of the spacesuit fabric and cooling tubes for use in estimating astronaut exposures. Finally it would be desirable to study the electron transmission characteristics for which the basic physical description of the fabric should be similar. An appropriate electron beam for experimental testing is being investigated. 
Secondly, upon complete understanding of the current spacesuit transmission properties we can improve estimates of the astronaut exposures in space activity [9]. In addition, this understanding of the performance of the fabric lay-up elements and experimental testing procedures will allow evaluation of alternate designs for improving the spacesuit design.

The next least protected critical organ is the lens of the eyes. They are protected mainly by the helmet components. Plans for testing the helmet component transmission properties are in progress.

\section{CONCLUSION}

Considerable progress has been made in understanding the basic transmission properties of the spacesuit fabric as it is now used in the space program. Some improved details for the physical model for the cooling tube are still required. It is desirable to also study the electron transmission properties to insure that a consistent picture of the spacesuit fabric is obtained. The basic fabric model as outlined is being integrated into a spacesuit model for estimates of astronaut exposures during future missions.

\section{REFERENCES}

1. Kosmos J.J., Nachtwey D.S., Hardy A. JSC/CTSDSS-241, 1-24-1989.

2. Wilson J. W. et al. NASA CP 3360, 1997.

3. Wilson, J. W. et al., Transport Methods and Interactions for Space Radiations. NASA Reference Publication, RP-1257, 1991

4. Heinbockel, J. H. et al. An Improved Neutron Transport Algorithm for Space Radiation. NASA TP2000-209865, 2000.

5. Clowdsley, M. S. et al., A comparison of the multigroup and collocation methods for solving the low energy neutron Boltzmann equation. Cana. J. Phys. 78: 45-56, 2000.

6. Wilson J.W., Tai H.A. NASA 2000-209864, 2000.

7. Tschalar C., Maccabee, H. D. Phys. Rev. B 1: 28632869, 1970.

8. Ross A.J. et al. NASA CP 3360, 1997.

9. Anderson B. M. et al. SAE 01ICES-2363, 2001.

\section{CONTACT}

The communicating author email address is:

John.W.Wilson@LaRC.NASA.GOV 